\title{
Emotion regulation and amygdala-precuneus connectivity: Focusing on attentional deployment
}

\author{
Jamie Ferri $^{1}$ - Joseph Schmidt ${ }^{2}$ - Greg Hajcak ${ }^{2}$. Turhan Canli ${ }^{2}$
}

Published online: 21 July 2016

(C) Psychonomic Society, Inc. 2016

\begin{abstract}
Attentional deployment is an emotion regulation strategy that involves shifting attentional focus. Deploying attention to non-arousing, compared to arousing, regions of unpleasant images has been associated with reduced negative affect, reduced amygdala activation, and increased activity in fronto-parietal control networks. The current study examined neural correlates and functional connectivity associated with using attentional deployment to increase negative affect (deploying attention towards arousing unpleasant information) or to decrease negative affect (deploying attention away from arousing unpleasant information), compared to naturally viewing unpleasant images, in 42 individuals while concurrently monitoring eye movements. Directing attention to both arousing and non-arousing regions resulted in enhanced fronto-parietal activation compared to natural viewing, but only directing attention to non-arousing regions was associated with changes in amygdala activation. There were no significant differences in connectivity between naturally viewing unpleasant images and focusing on arousing regions. However, naturally viewing unpleasant images, relative to focusing on non-arousing regions, was associated with increased connectivity between the amygdala and visual cortex, while focusing on non-arousing regions of unpleasant images, compared to natural viewing, was associated with increased connectivity between the amygdala and the precuneus. Amygdala-precuneus connectivity correlated positively with eye-tracking measures of attentional deployment success and
\end{abstract}

Jamie Ferri

jamie.ferri@ucsf.edu

1 Department of Psychiatry, University of California, San Francisco, CA 94143, USA

2 Stony Brook University, Stony Brook, NY 11794-2500, USA with trait reappraisal. Deploying attention away from arousing unpleasant information, then, may depend upon functional relationships between the amygdala and parietal regions implicated in attentional control. Furthermore, these relationships might relate to the ability to successfully implement attentional deployment, and the predisposition to utilize adaptive emotion regulation strategies.

Keywords Emotion $\cdot$ Attention $\cdot$ Functional connectivity

Attentional deployment is a common emotion regulation strategy that involves shifting attention towards or away from affective stimuli (Gross, 1998). For example, although individuals often find themselves captivated by emotionally salient scenes (e.g., car accidents), most individuals can turn their attention away from emotionally arousing stimuli (e.g., by physically looking away) and redirect attention elsewhere to complete a task or reduce emotion. Deploying attention away from emotional stimuli is effective in regulating affect across the lifespan (Mangelsdorf, Shapiro, \& Marzolf, 1995; Mather \& Carstensen, 2005) and is used even without explicit instruction or training (van Reekum et al., 2007). Whereas successful attentional deployment is associated with reduced negative affect in healthy individuals (Ferri, Schmidt, Hajcak, \& Canli, 2013; Johnson, 2009; Urry, 2010; Webb, Miles, \& Sheeran, 2012), deficits in attentional deployment have been observed in disorders such as anxiety and depression (Sears, Newman, Ference, \& Thomas, 2011; Wieser, Pauli, Weyers, Alpers, \& Mühlberger, 2009).

Despite the ubiquity and effectiveness of attentional deployment, neural models of emotion regulation have been primarily based on reappraisal - a cognitive strategy that involves changing the meaning of emotional stimuli in order to change emotional significance (Gross, 1998; Gross \& 
Thompson, 2007). These studies typically examine how reappraisal can be used to reduce (down-regulate) or increase (upregulate) affective experience in relation to natural viewing (Diers, Weber, Brocke, Strobel, \& Schönfeld, 2014). Compared to natural viewing, both down- and up-regulation of emotion via reappraisal is typically associated with increased activity in lateral and medial prefrontal regions including the middle frontal gyrus and the dorsomedial prefrontal cortex, as well as, to a lesser extent, increased activity in inferior parietal regions and the precuneus (for a review see Ochsner, Silvers \& Buhle, 2012). Down-regulation has been associated with corresponding decreased activity in the amygdala (Ochsner, Bunge, Gross \& Gabrieli, 2002), and up-regulation is correspondingly associated with increased activation in amygdala (Ochsner et al., 2004). Greater coupling between the amygdala and portions of the prefrontal cortex, including the orbital frontal and dorsomedial prefrontal cortex, has been associated with lower self-reported negative affect (Banks, Eddy, Angstadt, Nathan, \& Phan, 2007; Wager, Davidson, Hughes, Lindquist, \& Ochsner, 2008). These studies have culminated in a model suggesting that emotion regulation is achieved through regulation of the amygdala via activation of prefrontal regions associated with cognitive control, most commonly the middle, inferior, and superior frontal gyri and the dorsomedial prefrontal cortex (Ochsner \& Gross, 2008; Ochsner, Silvers, \& Buhle, 2012).

Fewer studies have investigated the neural mechanisms associated with attentional deployment, and these studies have yet to be thoroughly incorporated into emotion regulation models. We recently suggested that deploying attention within unpleasant pictures involves interactions between the amygdala and frontoparietal control regions that had been previously associated with reappraisal, including the middle frontal gyrus and the inferior parietal cortex (Ferri et al., 2013). We demonstrated that, compared to directing one's gaze to the arousing regions of an unpleasant image, directing gaze to relatively non-arousing regions of the same image successfully reduced negative affect and reduced amygdala activation when compliance was monitored with eye-tracking. These findings suggested that successfully deploying visual attention away from unpleasant visual content may involve the topdown regulation of amygdala activation by parietal, as well as prefrontal, regions.

There is evidence suggesting that reappraisal and attentional deployment rely on shared neural mechanisms and that individuals use these strategies concurrently. Reappraisalrelated changes in the posterior prefrontal cortex, dorsolateral prefrontal cortex, and inferior parietal cortex have been interpreted to reflect selective attention and working memory processes necessary to direct and maintain attention on aspects of the environment or stimuli that are relevant to reappraisal (Ochsner, Silvers, \& Buhle 2012) - processes that are critical to attentional deployment. One study demonstrated that individuals who are instructed to use reappraisal simultaneously utilize attentional deployment; not only do they shift their gaze away from unpleasant arousing stimuli but these shifts in gaze account for a significant percentage of neural activation associated with reappraisal (van Reekum et al., 2007). The tendency to use reappraisal has also been related to reductions in amygdala activation as well as increases in the middle frontal gyrus and inferior and superior parietal lobe during the passive viewing of emotional images (Drabant, McRae, Manuck, Hariri, \& Gross, 2009) - regions related to the successful use of both trait reappraisal and attentional deployment. The neural basis of trait reappraisal, then, may be related to the neural basis of attentional deployment.

In the present study, we examined neural activation and functional connectivity associated with the regulation of emotional experience via attentional deployment by examining both up-regulation (focusing on arousing content versus natural viewing) and down-regulation (focusing on non-arousing content vs. natural viewing) while concurrently tracking eye movements. By selecting these contrasts, which are analogous to those typically reported in studies of reappraisal, these results can be more easily integrated into existing reappraisalfocused models of emotion regulation (Ochsner et al., 2012). Based on previous studies, we expected that attentional deployment would involve increases in frontoparietal regions associated with the effortful control of emotion, including the middle frontal gyrus, precuneus, and inferior and superior parietal lobes, when directing attention toward or away from arousing stimuli. However, we hypothesized that only directing attention away from arousing information would reduce negative affect and amygdala activation. Using the amygdala as a seed region, we hypothesized that directing attention to non-arousing portions of unpleasant images would result in increased task-dependent covariation between the amygdala and these same frontoparietal control regions involved in effortful emotion regulation relative to the natural viewing of unpleasant images. Because we believe attentional deployment and reappraisal may rely on shared neural mechanisms, we also predicted that connectivity between these regions would correlate positively with the successful implementation of attentional deployment in the concurrent task (focusing on non-arousing region when instructed), and with trait use of reappraisal.

\section{Method}

\section{Participants}

Fifty-one healthy adults with no history of neurological or psychological illness were recruited to take part in a functional magnetic resonance imaging (fMRI) study of emotion and attention. History of neurological or psychological illness 
was assessed through phone screening prior to participation. Participants were excluded from the present analyses if they spent less than $50 \%$ of dwell time (cumulative fixation duration) in the focus region on focus trials $(n=4)$ : of those who did, one subject reported intentionally looking off-screen to avoid unpleasant images and three subjects closed their eyes during large portions of all trials due to self-reported sleepiness. Subjects were also excluded if they had motion in excess of $1 \mathrm{~mm}$ in any direction $(n=5)$. As a result, 42 individuals (24 women) with a mean age of 21.8 years $(S D=3.62)$ were included in the present analyses. Other contrast data from these participants were previously presented by Ferri and colleagues (2013) in Study 2 to investigate a separate set of hypotheses regarding direct comparisons between arousing and non-arousing conditions; current whole brain analyses, connectivity analyses, and correlations with behavioral measures have not been reported previously. The Committee on Research Involving Human Subjects at Stony Brook University approved this study and all participants provided informed consent and received payment for their participation. All participants were right-handed native English speakers with normal or corrected-to-normal vision.

\section{Stimuli}

A total of 100 images from the International Affective Picture System (Lang, Bradley, \& Cuthbert, 2008) were used in this study. Sixty unpleasant and 40 neutral images were used. Unpleasant images were rated as more arousing $(M=6.05$, $S D=2.25)$ than neutral images $(M=2.91, S D=1.92)$ and less pleasant $(M=2.28, S D=1.47)$ than neutral images $(M=5.14$, $S D=1.28$ ). For all unpleasant images, an arousing region and a non-arousing region were designated. For all neutral images, a non-arousing region was designated. Each participant naturally viewed 20 unpleasant images without modification (unpleasant no focus condition) and 20 neutral pictures were presented without a modification (neutral no focus condition). For the remaining 60 images, a blue circle was placed over a portion of the image. During the unpleasant arousing focus condition, the blue circle was placed over an arousing region of the unpleasant image. During the unpleasant non-arousing condition, the blue circle was placed over a non-arousing region of the unpleasant image. During the neutral non-arousing focus condition, the blue circle was placed over a nonarousing but visually complex portion of a neutral image. A figure depicting example images for each condition is illustrated in Fig. 1. Images were presented in 20 counterbalanced blocks, each $20 \mathrm{~s}$ in length, over the course of two 10-min long runs. Pictures were counterbalanced across subjects to ensure that unpleasant images appeared in the no focus, non-arousing focus, and arousing focus conditions an equal number of times and that neutral images appeared in the no focus and nonarousing focus conditions an equal number of times. The presentation of individual images was balanced to ensure that the average distance between the centers of focus regions across conditions did not differ.

The task was programmed using Experiment Builder software package (SR Research Ltd, version 1.10.165) and was designed to collect simultaneous eye-tracking and fMRI data. Stimuli were presented using an MRI-compatible $60 \mathrm{~Hz}$ projector with a $1,024 \times 768$ resolution that were back projected onto a mirror attached to the head coil.

\section{Procedure}

Participants were informed that they would view sets of images, and that before each set of images they would receive instructions to either freely view the images as they naturally would or to direct their eyes and attention only to a small portion of the image within a blue circle. Instructions to "look freely" or "look only at the part of the image in the blue circle" were presented for $5 \mathrm{~s}$ at the beginning of each block. Participants then viewed four images of the same type for $5 \mathrm{~s}$ each, for a total of $20 \mathrm{~s}$ per condition. Following each block of images, participants were allotted $5 \mathrm{~s}$ to rate the intensity of their negative affect on a scale of $1-4$, and then viewed a fixation screen for $20 \mathrm{~s}$. Each block therefore included an instruction period ( $5 \mathrm{~s})$, a set of four images ( $20 \mathrm{~s})$, and an affect rating period ( $5 \mathrm{~s})$, followed by rest (20 s).

\section{Eye-tracking data acquisition and analysis}

Eye position was sampled at $1,000 \mathrm{~Hz}$, using a long-range mounted EyeLink 1,000 MRI compatible eye-tracker. Eye position was mapped to screen coordinates using a 13-point calibration routine at the start of each experimental run. Calibrations were considered acceptable when the average error was equal to or less than $0.49^{\circ}$ and the maximum error was equal to or less than $0.99^{\circ}$ for each participant.

Data analysis was conducted using the DataViewer software package (SR Research Ltd, version 1.11.1). For focus trials, cumulative fixation duration (i.e., dwell time) within the blue circle was computed. During natural viewing trials, time spent in both non-arousing and arousing interest areas was calculated.

\section{fMRI data acquisition and analysis}

Data were acquired on a 3 Tesla Siemens TrioTim whole body scanner (Siemens Medical, Erlangen, Germany) with a 12channel head coil. An EPI sequence was used to collect 400 T2-star weighted whole-brain volumes with the following parameters: $\mathrm{TR}=2,500 \mathrm{~ms}, \mathrm{TE}=30 \mathrm{~ms}$, flip angle $=90^{\circ}$, matrix dimensions $=64 \times 64$, field of view $(\mathrm{FOV})=256$, $256 \mathrm{~mm}$, slices $=34$, aligned to the AC-PC, slice thickness $=4 \mathrm{~mm}$, slice acquisition $=$ interleaved, gap $=0$. 

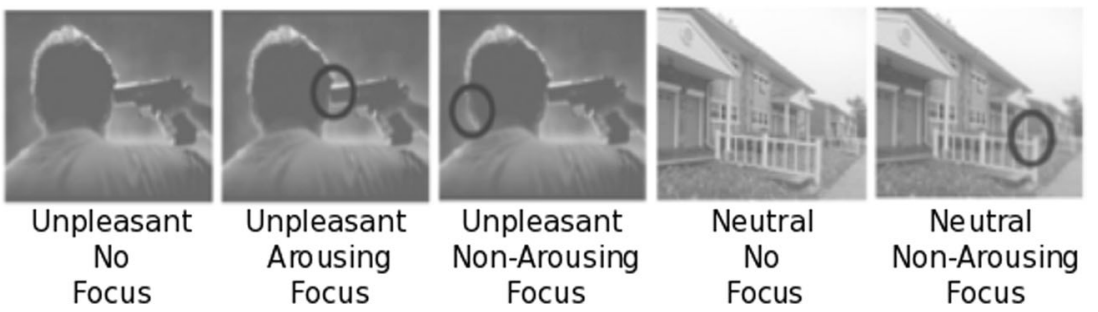

Fig. 1 A task schematic depicting example stimuli for each of the five conditions in the study: unpleasant no focus, unpleasant arousing focus, unpleasant non-arousing focus, neutral no focus, and neutral non-arousing focus

Image preprocessing Standard preprocessing procedures were performed in SPM8 starting with slice time correction, followed by realignment to the first volume in the image for motion correction. Realigned images were then normalized to standard Montreal Neurological Institute space, and spatially smoothed using a Gaussian kernel with 6 mm FWHM.

At the first level, single-subject SPMs were created for each subject from a model that specified the onsets and duration of the instruction period ( $5 \mathrm{~s}$ ), each condition (20 s), and the affect-rating period ( $5 \mathrm{~s}$ ). For each participant, across both runs, contrasts and reverse contrasts were created for unpleasant no focus-unpleasant non-arousing focus and unpleasant no focus-unpleasant arousing focus. In addition, the following contrasts were created to perform a conjunction analysis to determine coordinates for an amygdala seed region for psychophysiological interaction (PPI) analysis: unpleasant no focus-neutral no focus, unpleasant arousing focus-neutral nonarousing focus, and unpleasant non-arousing focus-neutral non-arousing focus. No additional regressors were included. The design matrix was convolved with the canonical HRF. Results for the unpleasant arousing focus and unpleasant non-arousing focus contrast were reported elsewhere (Ferri et al., 2013).

Image analysis At the second level, random effects analyses were conducted to test for statistical differences between conditions of interest using a one-sample $t$-test with contrasts created for each individual at the first level. Group-based (second-level) random effects analyses were then conducted on contrast images to test for statistically significant differences between conditions of interest using one-sample $t$-tests. In accordance with recommended practices for using clusterextent based thresholding (Woo, Krishnan, \& Wager, 2014), whole brain maps of these statistical tests were thresholded using an initial voxel-level cluster-defining uncorrected threshold of $p<.001$ (extent $=10$ ). Clusters were considered significant only if they reached a cluster-level Family-Wise Error (FWE) corrected threshold of $p<.05$.

Determination of seed regions Conjunction analysis was used to identify coordinates in the left and right amygdala that were responsive to affective stimuli. To determine the coordinates for the right and left amygdala, a conjunction analysis was performed across all unpleasant conditions compared to baseline (unpleasant no focus-neutral no focus, unpleasant arousing focus-neutral non-arousing focus, and unpleasant non-arousing focus-neutral non-arousing focus). The AAL atlas in WFU PickAtlas was used to ensure that the resulting activation was limited to the amygdala, as it is anatomically defined. The center of activation for the right $(22,-4,-18)$ and left $(-22,-4,-22)$ amygdala was then used as coordinates for the seed region for two separate functional connectivity analyses; one for the left amygdala, and one for the right. Both clusters were greater than 15 voxels in size, were significant after family-wise error (FWE) correction $(p<.05)$, and had $t$ values greater than 4.5. A 6-mm spherical region of interest (ROI) (equivalent to the smoothing kernel) surrounding those coordinates was then used to generate the time series for the physiological term.

Analysis of functional connectivity Task-related functional connectivity was assessed using PPI analysis (Friston et al., 1997). PPI analysis identifies activity in a given brain region that is explained by an interaction between the influence of activity in another area (physiological) and the experimental design (psychological). PPI analysis compares the functional relationship between regions across different conditions or tasks. A significant PPI interaction indicates that the contribution from one area to another changes significantly as a function of task condition. PPI analysis requires a design matrix which contains: (1) a psychological variable which represents the experimental design, (2) the time series of a given seed/source region, and (3) the interaction term. The interaction term represents the covariance in activity between the source region and the region that is significantly different between task conditions. A significant PPI effect, consequently, does not provide any information regarding the directional nature of the regression slopes under individual conditions it simply shows if covariation increases or decreases between the different task conditions (Friston, 1998; Friston et al., 1997). Functional connectivity was analyzed across different task conditions on a single subject level: Unpleasant no focus versus unpleasant arousing focus, and unpleasant no focus versus unpleasant non-arousing focus.

Each contrast was analyzed separately using the contrast [1 $\begin{array}{ll}0 & 0]\end{array}$ in which the first column represents the interaction term. 
The single subject contrast images were then entered into the second level random effects analysis using a one-sample $t$-test in order to identify different patterns of interaction as a function of task-dependent effects. A significant PPI effect indicates that the covariance between the source region (the amygdala) and another region is significantly higher in one condition than another (e.g., unpleasant arousing focus compared to unpleasant non-arousing focus). Clusters were considered significant if they reached a cluster-level FWE corrected threshold of $p<.05$.

We chose PPI analysis, using the amygdala as a seed region, in order identify task-dependent covariation between the amygdala and the remainder of the brain during up- and down-regulation of emotion via attentional deployment.

\section{Emotion regulation questionnaire}

Trait reappraisal was assessed using the Emotion Regulation Questionnaire (ERQ; Gross \& John, 2003). The ERQ is a 10item questionnaire that assesses the use of reappraisal and suppression to regulate emotion in everyday life. Items are rated on a 7-point scale from 1 (strongly disagree) to 7 (strongly agree). Internal consistency of the ERQ (alpha of .77 to .82 for reappraisal and .68 to .76 for suppression) is moderate to high.

\section{Analysis of relationship between functional connectivity, emotion regulation, and eye-tracking data}

To examine the relationship between the eye-tracking data, trait reappraisal, and functional connectivity, regions with greater task-related connectivity to the amygdala were extracted from SPM as eigenvariates and imported into IBM SPSS Statistics 19 (SPSS Inc., Chicago, IL, USA). Given the directional nature of our hypotheses, one-tailed Pearson's product correlations were conducted, controlling for multiple comparisons using false discovery rate (FDR). To assess relationships between amygdala-precuneus connectivity and visual compliance, activation from regions indicating greater connectivity with the amygdala during the unpleasant non-arousing $>$ unpleasant no focus condition was extracted and correlated with mean time spent in the non-arousing region as instructed across all nonarousing focus trials. Similarly, activation from regions indicating greater connectivity with the amygdala during the unpleasant non-arousing $>$ unpleasant no focus condition was extracted and correlated with self-reported scores on the ERQ to assess relationships between reappraisal, suppression, and connectivity. A correlation between reappraisal and time spent in non-arousing region during unpleasant non-arousing focus trials was then conducted to ensure relationships with connectivity variables were independent.

\section{Results} Whole brain analysis: unpleasant no focus vs. unpleasant
arousing focus

Naturally viewing unpleasant images, compared to focusing on an arousing portion of the image, resulted in enhanced activation in a cluster over bilateral occipital cortex, clusters over bilateral putamen/caudate and a cluster over bilateral cerebellum. Activations for this contrast are presented in Table 1 and Fig. 2.

Focusing on an arousing region, compared to naturally viewing an unpleasant image, resulted in enhanced activation in bilateral clusters over parietal regions consisting of inferior and superior parietal lobes and precuneus. In addition, focusing on an arousing region was associated with increased activation in bilateral clusters over middle frontal gyrus. Activations for this contrast are presented in Table 1 and Fig. 2.

\section{Whole brain analysis: unpleasant no focus versus unpleasant non-arousing focus}

Naturally viewing unpleasant images, relative to directing attention to a non-arousing portion of the image, resulted in a large cluster of activation centered over the bilateral visual cortex that also extended into the cerebellum, as well as into the parietal lobe, the parahippocampal gyrus, and the amygdala. An ROI analysis indicated significant activation in the left and right amygdala. Activations for this contrast are presented in Table 2 and Fig. 3.

Focusing on a non-arousing region of an unpleasant image, compared to natural viewing, resulted in activation in clusters over the right and left inferior/superior parietal lobe and precuneus, as well as clusters over the left and right middle/ superior frontal gyrus, and the left precentral gyrus. Activations for this contrast are presented in Table 2 and Fig. 3.

In sum, focusing on arousing and non-arousing regions of unpleasant images both elicit activation in bilateral inferior and super parietal lobes, precuneus, and middle frontal gyrus compared to free viewing unpleasant images, suggesting the act of focusing recruits similar regions regardless of the content of focus. However, only focusing on a non-arousing region resulted in reduced amygdala activation compared to free viewing.

\section{Functional connectivity: unpleasant no focus versus unpleasant arousing focus}

There were no differences in functional connectivity when comparing unpleasant images without a focus to unpleasant 
Table 1 Regions of the brain that were more active during the unpleasant no focus compared to unpleasant arousing focus conditions (top), and regions of the brain that were more active during the unpleasant arousing focus compared to unpleasant no focus conditions

\begin{tabular}{|c|c|c|c|c|}
\hline Region & Cluster size & $\begin{array}{l}\text { Peak } \\
\text { X Y Z coordinate }\end{array}$ & $\mathrm{t}^{*}$ & $p$ \\
\hline \multicolumn{5}{|l|}{ Unpleasant no focus $>$ unpleasant arousing focus } \\
\hline B occipital lobe/cuneus & 2,463 & $-3-859$ & 17.56 & $>.0001$ \\
\hline L putamen/caudate & 44 & -141618 & 5.02 & .031 \\
\hline B cerebellum & 111 & $-14-50-39$ & 4.82 & $>.0001$ \\
\hline $\mathrm{R}$ putamen/caudate & 64 & 162015 & 4.8 & .007 \\
\hline \multicolumn{5}{|l|}{ Unpleasant arousing focus $>$ unpleasant no focus } \\
\hline L inferior parietal lobe/precuneus & 662 & $-37-4341$ & 8.91 & $>.0001$ \\
\hline L precentral gyrus/middle frontal gyrus & 149 & $-48-134$ & 7.46 & $>.0001$ \\
\hline $\mathrm{R}$ inferior/superior parietal lobe/precuneus & 667 & $33-4449$ & 7.45 & $>.0001$ \\
\hline $\mathrm{R}$ middle frontal gyrus & 107 & $26-460$ & 5.8 & $>.0001$ \\
\hline
\end{tabular}

All activations were identified using a height threshold of $p<.001$ uncorrected and clusters are considered significant if they have a cluster level significance of $p<.05$, Family-Wise Error (FWE) corrected. X Y and Z coordinates are listed in Talairach space for the peak voxel within each cluster $\mathrm{B}=$ bilateral, $\mathrm{R}=$ right, $\mathrm{L}=$ left.

* The $t$ value indicates the peak statistical value for the cluster

images with an arousing focus using right or left amygdala as the seed region.

\section{Functional connectivity: unpleasant no focus versus unpleasant non-arousing focus}

Naturally viewing unpleasant images compared to focusing on a non-arousing region resulted in enhanced connectivity between the right amygdala and clusters over bilateral occipital cortex and right fusiform gyrus. Similarly, naturally viewing unpleasant images resulted in enhanced functional connectivity between the left amygdala and a large cluster over bilateral occipital cortex that extended into fusiform gyrus. These results are presented in Table 3 and Fig. 4.

Conversely, focusing on a non-arousing region, compared to naturally viewing unpleasant images, resulted in enhanced connectivity between central and posterior portions of the precuneus and both left and right amygdala. These results are presented in Table 4 and Fig. 4.

\section{Eye-tracking}

During natural viewing of unpleasant images, participants spent an average of $1,398.12 \mathrm{~ms}(\mathrm{SD}=287.77)$ fixating in the arousing region and $207.94(\mathrm{SD}=117.12)$ fixating in the nonarousing region. When asked to focus on an arousing region, participants spent an average of $3,330.45 \mathrm{~ms}(\mathrm{SD}=274.50)$ fixating in the arousing region as instructed, and $16.58 \mathrm{~ms}$ $(\mathrm{SD}=17.33)$ fixating in the non-arousing region. When asked to focus on a non-arousing region, participants spent an average of 3,183.98 $\mathrm{ms}(\mathrm{SD}=326.91)$ in the non-arousing region as instructed and $127.20 \mathrm{~ms}(\mathrm{SD}=99.62)$ in the arousing region. Time spent in arousing and non-arousing regions for each of the unpleasant conditions is illustrated in Fig. 5. Participants spent
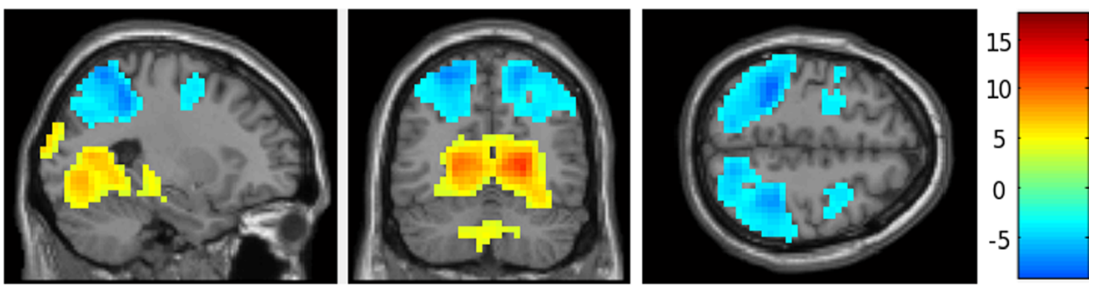

$$
X=-26, Y=-60, Z=52
$$

Fig. 2 Regions of the brain that were more active during the unpleasant no focus > unpleasant arousing focus condition are depicted in red; regions of the brain that were more active during the unpleasant arousing focus $>$ unpleasant no focus condition are depicted in blue. Color bars represent $t$ values; higher values are displayed in warmer colors. Results were identified using a height threshold of $p<.001$ uncorrected and clusters are considered significant if they have a cluster level significance of $p<.05$, family-wise error (FWE) corrected. $\mathrm{X}=-26$, $Y=-60, Z=52$ 
Table 2 Peak activations for unpleasant no focus compared to unpleasant non-arousing focus conditions (top), and regions of the brain that were more active during the unpleasant non-arousing focus compared to unpleasant no focus conditions

\begin{tabular}{llll}
\hline Region & Cluster size & $\begin{array}{l}\text { Peak } \\
\text { X Y Z coordinate }\end{array}$ & $t^{*}$ \\
\hline Unpleasant no focus $>$ unpleasant non-arousing focus & & & \\
B occipital lobe/cuneus & 3,944 & $-7-855$ & 15.51 \\
L amygdala (ROI analysis) & 17 & $-22-8-14$ & 5.93 \\
R amygdala (ROI analysis) & 5 & $26-8-14$ & 4.28 \\
Unpleasant non-arousing focus $>$ unpleasant no focus & & 11.32 \\
R inferior parietal lobe/precuneus & 880 & $45-5038$ & 7.93 \\
L superior parietal lobe/precuneus & 705 & $-19-6757$ & 7.31 \\
R middle frontal gyrus & 644 & 344615 & 5.81 \\
L middle frontal gyrus & 77 & -364313 & $>.0001$ \\
L precentral gyrus & 47 & $-33-1344$ & .0001 \\
\hline
\end{tabular}

All activations were identified using a height threshold of $p<.001$ uncorrected and clusters are considered significant if they have a cluster level significance of $p<.05$, Family-Wise Error (FWE) corrected. X Y and Z coordinates are listed in Talairach space for the peak voxel within each cluster $\mathrm{B}=$ bilateral, $\mathrm{R}=$ right, $\mathrm{L}=$ left, $\mathrm{ROI}=$ region of interest

* The $t$ value indicates the peak statistical value for the cluster.

significantly more time in the arousing region when instructed to do so compared to the non-arousing region when instructed to do so $(t(40)=4.53, p>.001)$.

\section{Correlations between amygdala-precuneus connectivity and visual compliance}

Individuals who spent more time in the non-arousing region as instructed were characterized by greater connectivity between the right (but not left) amygdala and the precuneus during the unpleasant non-arousing focus condition, relative to the unpleasant no focus condition (right: $r=.32, p=.022$; left: $r=.16, p=.157$ ).

\section{Correlations between amygdala-precuneus connectivity and trait reappraisal}

Individuals who reported high levels of trait reappraisal also had greater connectivity between the precuneus and right $(r=$
$.36, p=.011)$ and left $(r=.39, p=.005)$ amygdala during the unpleasant non-arousing focus condition relative to the unpleasant no focus condition. However, trait suppression was not significantly related to connectivity between precuneus and right $(r=-.19, p=.120)$ or left $(r=.17, p=.114)$ amygdala. Trait reappraisal was not significantly related to the time spent in the non-arousing region as instructed $(r=.15, p=$ .184).

\section{Discussion}

The current study examined neural activation and functional connectivity associated with regulation of emotional experience by way of attentional deployment. Furthermore, this study examined correlations between functional connectivity and both occulomotor measures of attentional deployment success and self-reported trait reappraisal.
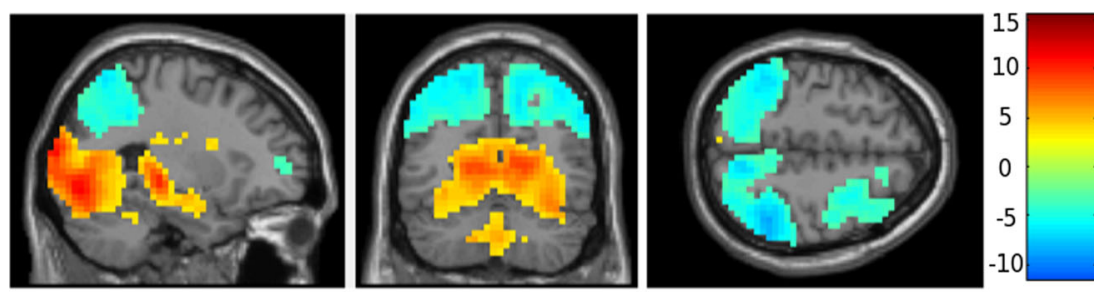

$$
X=-26, Y=-60, Z=52
$$

Fig. 3 Regions of the brain that were more active during the unpleasant no focus > unpleasant non-arousing focus condition are depicted in red; regions of the brain that were more active during the unpleasant nonarousing focus $>$ unpleasant no focus condition are depicted in blue. Color bars represent $t$ values; higher values are displayed in warmer colors. Results were identified using a height threshold of $p<.001$ uncorrected and clusters are considered significant if they have a cluster level significance of $p<.05$, family-wise error (FWE) corrected. $\mathrm{X}=-26$, $Y=-60, Z=52$ 
Table 3 Psychophysiological interaction (PPI) results using the right (top) and left (bottom) amygdala as a seed region for the unpleasant no focus compared to unpleasant non-arousing focus conditions

\begin{tabular}{|c|c|c|c|c|c|}
\hline & Region & Cluster size & $\begin{array}{l}\text { Peak } \\
\text { X Y Z coordinate }\end{array}$ & $t^{*}$ & $p$ \\
\hline \multirow[t]{2}{*}{$\mathrm{R}$ amygdala } & $\mathrm{R}$ middle temporal gyrus & 566 & $45-7414$ & 5.31 & $>.0001$ \\
\hline & R fusiform gyrus & 57 & $38-41-15$ & 4.88 & $>.0001$ \\
\hline \multirow[t]{3}{*}{ L amygdala } & L cerebellum & 252 & $-36-64-11$ & 5.31 & $>.0001$ \\
\hline & $\mathrm{R}$ fusiform gyrus & 176 & $30-45-12$ & 4.57 & $>.0001$ \\
\hline & $\mathrm{R}$ middle temporal gyrus & 84 & $45-7414$ & 4.38 & $>.0001$ \\
\hline
\end{tabular}

All activations were identified using a height threshold of $p<.001$ uncorrected and clusters are considered significant if they have a cluster level significance of $p<.05$, Family-Wise Error (FWE) corrected. X Y and Z coordinates are listed in Talairach space for the peak voxel within each cluster $\mathrm{B}=$ bilateral, $\mathrm{R}=$ right, $\mathrm{L}=$ left

* The $t$ value indicates the peak statistical value for the cluster

We observed increased activation in the middle frontal gyrus and inferior and superior parietal regions regardless of whether participants' gaze was directed at the arousing or non-arousing region of an unpleasant image (relative to natural viewing), corresponding to up- or down-regulation of negative affect, respectively. These results are akin to studies demonstrating that both increasing and decreasing negative affect via reappraisal is associated with increased activation in the posterior prefrontal cortex, dlPFC, and inferior parietal regions (Eippert et al., 2007; Kim \& Hamann, 2007; Ochsner et al., 2004; van Reekum et al., 2007). These reappraisalrelated increases in posterior regions of the prefrontal cortex and inferior parietal regions have been associated with selective attention and working memory demands associated with reappraisal (Ochsner, Silvers, \& Buhle, 2012). Studies of cognitive distraction have also reported increases in these regions compared to natural viewing, but these studies have only investigated down-regulation of affect (Kanske, Heissler, Schönfelder, Bonger, \& Wessa, 2011; McRae et al., 2010). In a previous manuscript based on subjects from the current study we demonstrated activation in these regions as a general result of focus (across neutral and unpleasant conditions); however, relatively greater activation was seen in these regions when focusing on a non-arousing compared to an arousing region of an unpleasant image, suggesting activations in these regions do not simply reflect task-based attentional demands (Ferri et al., 2013). Findings from our current study suggest that simply directing visual gaze to more or less arousing regions of an unpleasant image recruits control regions in a similar fashion to utilizing more widely studied cognitive emotion regulation strategies to up- or down-regulate affective experiences (Ochsner, Silvers, \& Buhle, 2012). Despite changes in the middle frontal gyrus and inferior/superior parietal regions and the precuneus being common to both upand down-regulation, we only observed significant changes in amygdala activation when participants directed their gaze to non-arousing regions of unpleasant images.
We also observed task-dependent changes in connectivity between the amygdala and the precuneus when participants focused on a non-arousing region compared to freely viewing unpleasant images. Strikingly, the degree of amygdala-toprecuneus connectivity correlated with task compliance: Individuals who stayed on the focus region as instructed had greater interregional coactivation between the amygdala and the precuneus. These results complement studies of reappraisal and distraction (Kanske et al., 2011; Urry et al., 2006), which have also demonstrated that down-regulation of emotion is associated with increased connectivity between amygdala and prefrontal and parietal control regions in the parietal cortex, including the precuneus, as well as prefrontal regions such as the dorsomedial and dorsolateral prefrontal cortex. Our task simply involves orienting and maintaining attention away from emotional content and only demonstrated enhanced connectivity between the amygdala and the precuneus compared to natural viewing. The region of the precuneus observed in this study overlapped with previous reports of precuneus-amygdala connectivity during distraction (Kanske et al., 2011), and corresponded to central and posterior subdivisions of the precuneus that have been associated with executive function, working memory, motor planning, and visual control in both humans and monkeys (Bzdok et al., 2015; Margulies et al., 2009). Specifically, this region of the precuneus has been considered part of the dorsal "where" pathway of visual processing (Ungerleider \& Mishkin, 1982), which is associated with directing attention in space, visuomotor planning, and the analysis of visuospatial movement (Goodale \& Milner, 1992; Goodale, 2008). Together these findings suggest that this central posterior region of the precuneus may play an important role in the successful implementation of attentional deployment, particularly visual attentional deployment. Importantly, while frontal and parietal activations were a general effect across unpleasant arousing and non-arousing image, increased connectivity between the precuneus and the amygdala demonstrates specificity to 


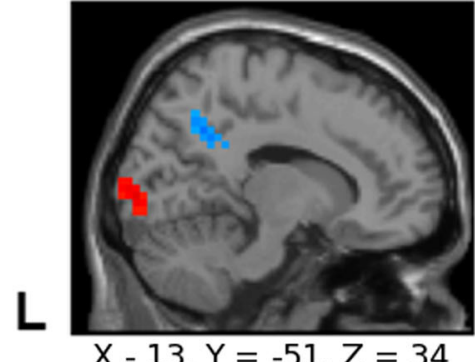

$X-13, Y=-51, Z=34$
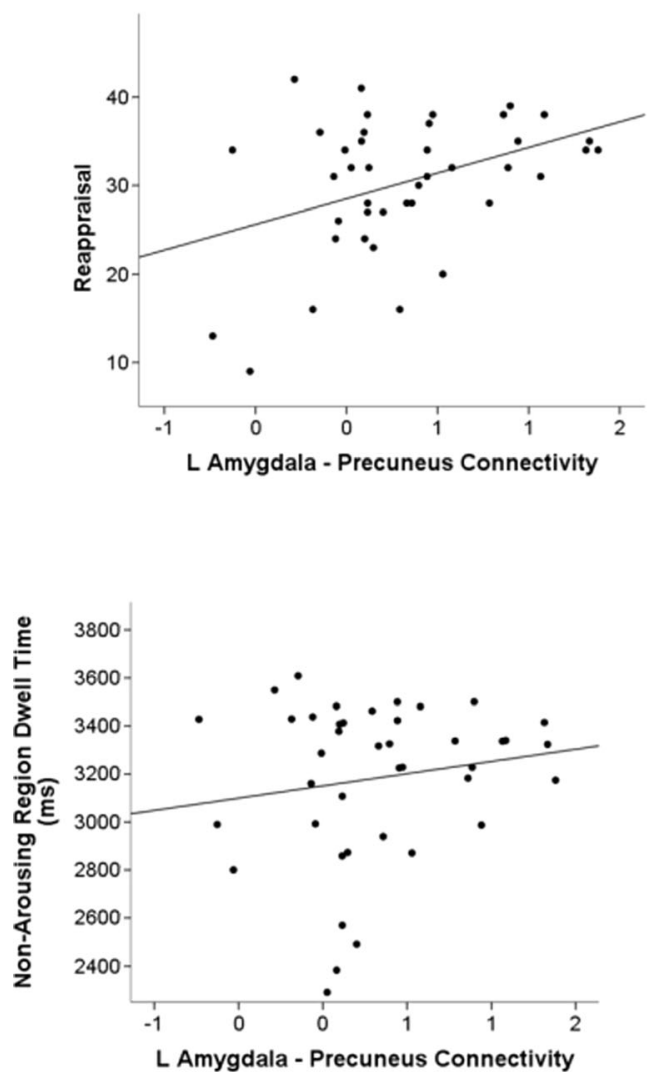

Fig. 4 Regions of the brain with greater functional connectivity with the amygdala during the unpleasant no focus $>$ unpleasant non-arousing focus condition are depicted in red, regions of the brain with greater connectivity with the amygdala during the unpleasant non-arousing focus $>$ unpleasant no focus condition are depicted in blue. Results

directing attention to the non-arousing region of an unpleasant image.

The precuneus has previously been associated with visual attention and inhibitory control (Hopfinger, Buonocore, \& Mangun, 2000; Pessoa, Kastner, \& Ungerleider, 2003) and may consequently play an important role in redirecting and maintaining attention on goal-relevant stimuli during emotion regulation. Both functional and structural relationships between the amygdala and the precuneus have been reported previously. For example, direct anatomical relationships between parietal regions and the amygdala have been observed in animal-tracing studies (Leichnetz, 2001; Parvizi, Van Hoesen, Buckwalter, \& Damasio, 2006). In healthy human
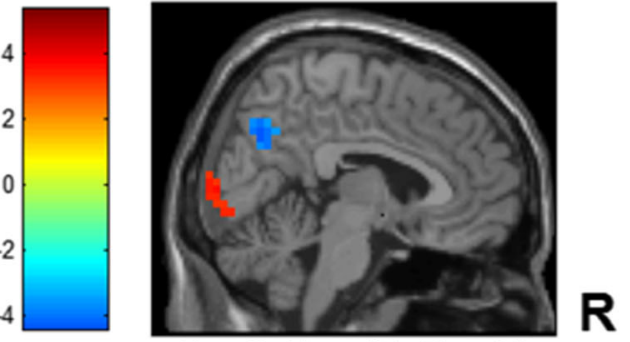

$X=6, Y=-64, Z=33$
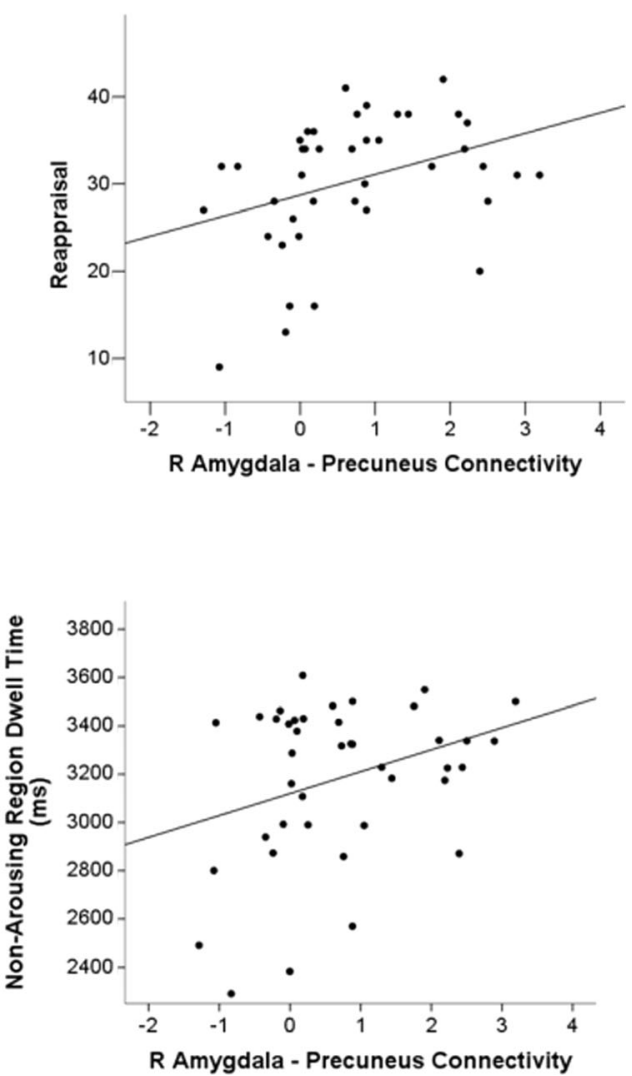

were identified using a height threshold of $p<.001$ uncorrected and clusters are considered significant if they have a cluster level significance of $p<.05$, family-wise error (FWE) corrected. Left: $\mathrm{X}=$ 13, $Y=-51, Z=34$, Right: $X=6, Y=-64, Z=33$

populations, negative connectivity has consistently been observed at rest between the amygdala and regions related to cognitive processes and the effortful regulation of affect, including the precuneus (Roy et al., 2009; Zhang \& Li, 2012). Disrupted connectivity between the precuneus and the amygdala has been reported in psychiatric populations, including children diagnosed with bipolar disorder (Rich et al., 2008; Stoddard et al., 2015), adolescent depression (Cullen et al., 2014), adults with childhood maltreatment (van der Werff et al., 2013), adults with the dissociative subtype of posttraumatic stress disorder (Nicholson et al., 2015), and individuals with schizophrenia (Mukherjee et al., 2012). Taken together, these findings suggest that the precuneus may 
Table 4 Psychophysiological interaction (PPI) results using the right (top) and left (bottom) amygdala as a seed region for the unpleasant nonarousing focus compared to unpleasant no focus conditions

\begin{tabular}{|c|c|c|c|c|c|}
\hline & Region & Cluster size & $\begin{array}{l}\text { Peak } \\
\text { X Y Z coordinate }\end{array}$ & $t^{*}$ & $p$ \\
\hline $\mathrm{R}$ amygdala & B precuneus & 48 & $4-6832$ & 3.95 & .007 \\
\hline $\mathrm{L}$ amygdala & R precuneus & 35 & $7-5733$ & 4.27 & .029 \\
\hline
\end{tabular}

All activations were identified using a height threshold of $p<.001$ uncorrected and clusters are considered significant if they have a cluster level significance of $p<.05$, Family-Wise Error (FWE) corrected. X Y and $\mathrm{Z}$ coordinates are listed in Talairach space for the peak voxel within each cluster

$\mathrm{B}=$ bilateral, $\mathrm{R}=$ right, $\mathrm{L}=$ left

${ }^{*}$ The $t$ value indicates the peak statistical value for the cluster

contribute to emotion regulation through attentional processes, such as orienting and maintaining attention away from emotional information, and that impaired connectivity may contribute to psychiatric disorders with social and emotional components. The current study suggests that the precuneus may play a direct role in the regulation of amygdala reactivity to emotional stimuli when emotion regulation involves shifts in attention.

In addition to being related to the successful implementation of attentional deployment, the degree of connectivity between the amygdala and the precuneus also correlated positively with trait reappraisal. Previous studies have shown that individual differences in trait reappraisal can impact the neural response to emotional images even in the absence of emotion regulation instructions: Women who scored higher on trait reappraisal showed reduced amygdala activation and increased activation in prefrontal and parietal regions during passive viewing of unpleasant images (Drabant et al., 2009). Furthermore, among depressed women anticipating negative images, trait reappraisal correlated negatively with amygdala activation (Abler, Erk, Herwig, \& Walter, 2007). Finally,

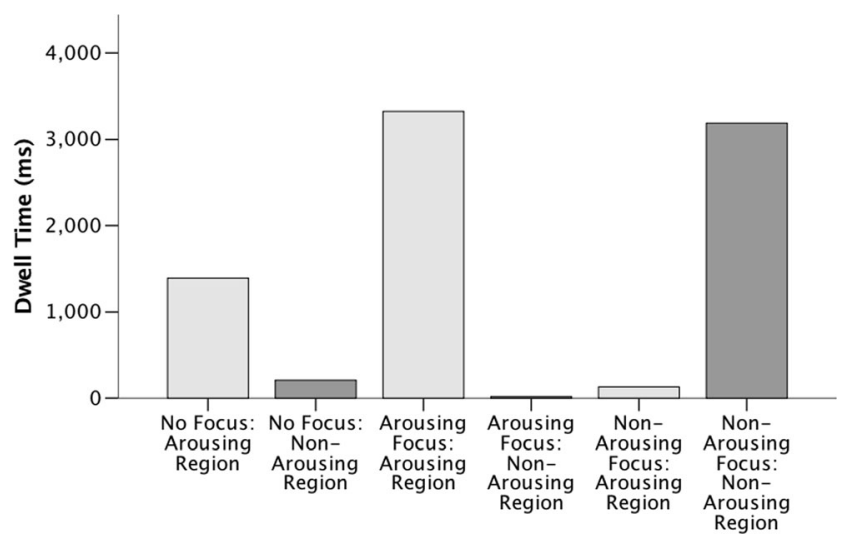

Fig. 5 A bar graph depicting the time spent in the arousing and nonarousing focus regions for each of the three unpleasant conditions: unpleasant no focus, unpleasant arousing focus, and unpleasant nonarousing focus individuals who score higher on trait reappraisal demonstrate less amygdala activation when instructed to inhibit responses to sad faces (Vanderhasselt, Baeken, Van Schuerbeek, Luypaert, \& De Raedt, 2013). An inverse relation between amygdala versus frontal and parietal activation as a function of trait reappraisal has been interpreted to represent the spontaneous use of reappraisal during affective picture viewing (Drabant et al., 2009). In the current study, individuals who scored higher on a measure of trait reappraisal had higher amygdala-to-precuneus connectivity when directing attention to non-arousing regions, despite the fact that the current paradigm did not involve reappraisal. This suggests that greater functional connectivity supporting attentional deployment may relate to the tendency to use reappraisal in everyday life. Specifically, greater connectivity between the precuneus and the amygdala may be associated with improved attentional deployment, which could also facilitate reappraisal. This is consistent with previous work which demonstrated that individuals use attentional deployment concurrently with reappraisal (van Reekum et al., 2007), and with the interpretation of dorsolateral prefrontal, posterior prefrontal and inferior parietal activations during reappraisal as reflecting selective attention and working memory processes that are necessary for the successful use of reappraisal (Ochsner, Silvers, \& Buhle, 2012). However, further investigation is required to dissociate emotional reactivity from regulation, and to simultaneously assess multiple emotion regulation strategies.

The current study used PPI analysis to explore temporal correlations between the left and the right amygdala and spatially remote locations. Although this method was useful in identifying regions of the brain that covary with amygdala activation during visual attentional deployment in one task condition compared to another, it is not suited to simultaneously assess functional relationships between regions independent of the amygdala. In addition, this methodology only determines that the degree of covariation between these regions changes as a function of condition; this method does not provide information regarding the directional nature of regression slopes under individual condition. Future studies employing alternate methodologies would be needed to determine the directionality of influence and mechanistic relationships between these regions. Other hypothesis-driven approaches, such as dynamic causal modeling, may be better suited to infer causality in future studies.

In conclusion, the current work suggests that similar to reappraisal, using attentional deployment to either increase or decrease attention to arousing information results in enhanced activation of frontoparietal control regions including the middle frontal gyrus, regions of the parietal cortex including the precuenus and inferior and superior parietal lobe, compared to natural viewing. Conversely, only using attentional deployment to decrease attention to negative information resulted in significant changes in amygdala activation. The 
current findings also extend previous work on functional connectivity during cognitive emotion regulation (Banks et al., 2007; Kanske et al., 2011) by demonstrating that taskdependent coupling between the amygdala and central and posterior portions of the precuneus is uniquely increased during visual attentional deployment. Further, the strength of coupling between these regions predicts attentional deployment success, and individual differences in trait reappraisal. While increases in frontal and parietal regions were common to both unpleasant arousing and non-arousing conditions, these connectivity results demonstrate the importance of functional relationships between the precuneus and the amygdala specifically when orienting and maintaining attention away from arousing aspects of unpleasant stimuli - a functional relationship which may facilitate other cognitive forms of emotion regulation, including reappraisal.

Acknowledgments The authors declare no competing financial interests. This work was supported by funding from NSF BCS-0843346 (TC) and NIH T32-MHO9920 (Ford).

\section{References}

Abler, B., Erk, S., Herwig, U., \& Walter, H. (2007). Anticipation of aversive stimuli activates extended amygdala in unipolar depression. Journal of Psychiatric Research, 41(6), 511-522. doi:10.1016/j. jpsychires.2006.07.020

Banks, S. J., Eddy, K. T., Angstadt, M., Nathan, P. J., \& Phan, K. L. (2007). Amygdala-frontal connectivity during emotion regulation. Social Cognitive and Affective Neuroscience, 2(4), 303-312. doi:10.1093/scan/nsm029

Bzdok, D., Heeger, A., Langner, R., Laird, A. R., Fox, P. T., PalomeroGallagher, N., ... Eickhoff, S. B. (2015). Subspecialization in the human posterior medial cortex. Neuroimage, 106, 55-71.

Cullen, K. R., Westlund, M. K., Klimes-Dougan, B., Mueller, B. A., Houri, A., Eberly, L. E., \& Lim, K. O. (2014). Abnormal amygdala resting-state functional connectivity in adolescent depression. JAMA Psychiatry, 71(10), 1138-1147.

Diers, K., Weber, F., Brocke, B., Strobel, A., \& Schönfeld, S. (2014). Instructions matter: A comparison of baseline conditions for cognitive emotion regulation paradigms. Frontiers in Psychology, 5, 347. doi:10.3389/fpsyg.2014.00347

Drabant, E. M., McRae, K., Manuck, S. B., Hariri, A. R., \& Gross, J. J. (2009). Individual differences in typical reappraisal use predict amygdala and prefrontal responses. Biological Psychiatry, 65(5), 367-373. doi:10.1016/j.biopsych.2008.09.007

Eippert, F., Veit, R., Weiskopf, N., Erb, M., Birbaumer, N., \& Anders, S. (2007). Regulation of emotional responses elicited by threat-related stimuli. Human Brain Mapping, 28(5), 409-423. doi:10.1002/ hbm.20291

Ferri, J., Schmidt, J., Hajcak, G., \& Canli, T. (2013). Neural correlates of attentional deployment within unpleasant pictures. NeuroImage, 70 , 268-277. doi:10.1016/j.neuroimage.2012.12.030

Friston, K. J. (1998). Imaging neuroscience: Principles or maps? Proceedings of the National Academy of Sciences, 95(3), 796-802.

Friston, K. J., Buechel, C., Fink, G. R., Morris, J., Rolls, E., \& Dolan, R. J. (1997). Psychophysiological and modulatory interactions in neuroimaging. NeuroImage, 6(3), 218-229.
Goodale, M. A. (2008). Action without perception in human vision. Cognitive Neuropsychology, 25(7-8), 891-919.

Goodale, M. A., \& Milner, A. D. (1992). Separate visual pathways for perception and action. Trends in Neuroscience., 15(1), 20-25.

Gross, J. J. (1998). The emerging field of emotion regulation: An integrative review. Review of General Psychology, 2(3), 271-299. doi:10.1037/1089-2680.2.3.271

Gross, J. J., \& John, O. (2003). Individual differences in two emotion regulation processes: Implications for affect, relationships, and wellbeing. Journal of Personality and Social Psychology, 85, 348-362.

Gross, J. J., \& Thompson, R. A. (2007). Emotion regulation: Conceptual foundations. In J. J. Gross (Ed.), Handbook of emotion regulation (pp. 3-24). New York, NY US: Guilford Press.

Hopfinger, J. B., Buonocore, M. H., \& Mangun, G. R. (2000). The neural mechanisms of top-down attentional control. Nature Neuroscience, 3(3), 284-291.

Johnson, D. R. (2009). Goal-directed attentional deployment to emotional faces and individual differences in emotional regulation. Journal of Research in Personality, 43(1), 8-13.

Kanske, P., Heissler, J., Schönfelder, S., Bonger, A., \& Wessa, M. (2011). How to regulate emotion? Neural networks for reappraisal and distraction. Cerebral Cortex, 21(6), 1379-1388. doi:10.1093/ cercor/bhq216

Kim, S. H., \& Hamann, S. (2007). Neural correlates of positive and negative emoiton regulation. Journal of Cognitive Neuroscience, 19(5), 776-798.

Lang, P. J., Bradley, M. M., \& Cuthbert, B. N. (2008). International affective picture system (IAPS): Affective ratings of pictures and instruction manual. Technical Report A-8. Gainesville, FL: University of Florida.

Leichnetz, G. R. (2001). Connections of the medial posterior parietal cortex (area 7m) in the monkey. The Anatomical Record, 263(2), 215-236.

Mangelsdorf, S. C., Shapiro, J. R., \& Marzolf, D. (1995). Developmental and temperamental differences in emotional regulation in infancy. Child Development, 66(6), 1817-1828. doi:10.2307/1131912

Margulies, D. S., Vincent, J. L., Kelly, C., Lohmann, G., Uddin, L. Q., Biswal, B. B., ... Petrides, M. (2009). Precuneus shares intrinsic functional architecture in humans and monkeys. Proceedings of the National Academy of Sciences, 106(47), 20069-20074.

Mather, M., \& Carstensen, L. (2005). Aging and motivated cognition: The positivity effect in attention and memory. Trends in Cognitive Sciences, 9, 496-502.

McRae, K., Hughes, B., Chopra, S., Gabrieli, J. D. E., Gross, J. J., \& Ochsner, K. N. (2010). The neural bases of distraction and reappraisal. Journal of Cognitive Neuroscience, 22(2), 248-262.

Mukherjee, P., Whalley, H. C., McKirdy, J. W., McIntosh, A. M., Johnstone, E. C., Lawrie, S. M., \& Hall, J. (2012). Lower effective connectivity between amygdala and parietal regions in response to fearful faces in schizophrenia. Schizophrenia Research, 134(2-3), 118-124. doi:10.1016/j.schres.2011.09.033

Nicholson, A. A., Densmore, M., Frewen, P. A., Théberge, J., Neufeld, R. W. J., McKinnon, M. C., \& Lanius, R. A. (2015). The dissociative subtype of posttraumatic stress disorder: Unique resting-state functional connectivity of basolateral and centromedial amygdala complexes. Neuropsychopharmacology.

Ochsner, K. N., \& Gross, J. J. (2008). Cognitive emotion regulation: Insights from social cognitive and affective neuroscience. Current Directions in Psychological Science, 17(2), 153-158. doi:10.1111/ j.1467-8721.2008.00566.x

Ochsner, K. N., Bunge, S. A., Gross, J. J., \& Gabrieli, J. D. E. (2002). Rethinking feelings: An fMRI study of the cognitive regulation of emotion. Journal of Cognitive Neuroscience, 14(8), 1215-1229. doi:10.1162/089892902760807212

Ochsner, K. N., Ray, R. D., Cooper, J. C., Robertson, E. R., Chopra, S., Gabrieli, J. D. E., \& Gross, J. J. (2004). For better or for worse: 
Neural systems supporting the cognitive down- and up-regulation of negative emotion. NeuroImage, 23(2), 483-499.

Ochsner, K. N., Silvers, J. A., \& Buhle, J. T. (2012). Functional imaging studies of emotion regulation: A synthetic review and evolving model of the cognitive control of emotion. Annals of the New York Academy of Sciences, 1251, E1-E24. doi:10.1111/j.17496632.2012.06751.x

Parvizi, J., Van Hoesen, G. W., Buckwalter, J., \& Damasio, A. (2006). Neural connections of the posteromedial cortex in the macaque. Proceedings of the National Academy of Sciences of the United States of America, 103(5), 1563-1568.

Pessoa, L., Kastner, S., \& Ungerleider, L. G. (2003). Neuroimaging studies of attention: From modulation of sensory processing to top-down control. The Journal of Neuroscience, 23(10), 3990-3998.

Rich, B. A., Fromm, S. J., Berghorst, L. H., Dickstein, D. P., Brotman, M. A., Pine, D. S., \& Leibenluft, E. (2008). Neural connectivity in children with bipolar disorder: impairment in the face emotion processing circuit. Journal of Child Psychology and Psychiatry, and Allied Disciplines, 49(1), 88-96. doi:10.1111/j.14697610.2007.01819.x

Roy, A. K., Shehzad, Z., Margulies, D. S., Kelly, A. M. C., Uddin, L. Q., Gotimer, K., ... Milham, M. P. (2009). Functional connectivity of the human amygdala using resting state fMRI. Neuroimage, 45(2), 614-626. doi: 10.1016/j.neuroimage.2008.11.030

Sears, C. R., Newman, K. R., Ference, J. D., \& Thomas, C. L. (2011). Attention to emotional images in previously depressed individuals: An eye-tracking study. Cognitive Therapy and Research, 35(6), 517-528. doi:10.1007/s10608-011-9396-5

Stoddard, J., Hsu, D., Reynolds, R. C., Brotman, M. A., Ernst, M., Pine, D. S., ... Dickstein, D. P. (2015). Aberrant amygdala intrinsic functional connectivity distinguishes youths with bipolar disorder from those with severe mood dysregulation. Psychiatry Research: Neuroimaging, 231(2), 120-125.

Ungerleider, L. G., \& Mishkin, M. (1982). Two cortical visual systems. In D. J. Ingle, M. A. Goodale, \& R. J. W. Mansfield (Eds.), Analysis of visual behavior (pp. 549-586). Boston, MA: MIT Press.

Urry, H. L. (2010). Seeing, thinking, and feeling: Emotion-regulating effects of gaze-directed cognitive reappraisal. Emotion, 10(1), 125-135. doi:10.1037/a0017434
Urry, H. L., van Reekum, C. M., Johnstone, T., Kalin, N. H., Thurow, M. E., Schaefer, H. S., ... Davidson, R. J. (2006). Amygdala and ventromedial prefrontal cortex are inversely coupled during regulation of negative affect and predict the diurnal pattern of cortisol secretion among older adults. The Journal Of Neuroscience: The Official Journal Of The Society For Neuroscience, 26(16), 4415-4425.

van der Werff, S. J. A., Pannekoek, J. N., Veer, I. M., van Tol, M. J., Aleman, A., Veltman, D. J., ... van der Wee, N. J. A. (2013). Resting-state functional connectivity in adults with childhood emotional maltreatment. Psychological Medicine, 43(09), 1825-1836.

van Reekum, C. M., Johnstone, T., Urry, H. L., Thurow, M. E., Schaefer, H. S., Alexander, A. L., \& Davidson, R. J. (2007). Gaze fixations predict brain activation during the voluntary regulation of pictureinduced negative affect. NeuroImage, 36(3), 1041-1055.

Vanderhasselt, M.-A., Baeken, C., Van Schuerbeek, P., Luypaert, R., \& De Raedt, R. (2013). Inter-individual differences in the habitual use of cognitive reappraisal and expressive suppression are associated with variations in prefrontal cognitive control for emotional information: An event related fMRI study. Biological Psychology, 92(3), 433-439.

Wager, T. D., Davidson, M. L., Hughes, B. L., Lindquist, M. A., \& Ochsner, K. N. (2008). Prefrontal-subcortical pathways mediating successful emotion regulation. Neuron, 59(6), 1037-1050. doi:10.1016/j.neuron.2008.09.006

Webb, T. L., Miles, E., \& Sheeran, P. (2012). Dealing with feeling: A meta-analysis of the effectiveness of strategies derived from the process model of emotion regulation. Psychological Bulletin, 138(4), 775-808. doi:10.1037/a0027600

Wieser, M. J., Pauli, P., Weyers, P., Alpers, G. W., \& Mühlberger, A. (2009). Fear of negative evaluation and the hypervigilanceavoidance hypothesis: An eye-tracking study. Journal of Neural Transmission, 116(6), 717-723. doi:10.1007/s00702-008-0101-0

Woo, C.-W., Krishnan, A., \& Wager, T. D. (2014). Cluster-extent based thresholding in fMRI analysis: Pitfalls and recommendations. Neuroimage (91), 412-419.

Zhang, S., \& Li, C.-S. R. (2012). Functional connectivity mapping of the human precuneus by resting state fMRI. NeuroImage, 59(4), 35483562. doi:10.1016/j.neuroimage.2011.11.023 